\title{
Sputum versus bronchoscopy for diagnosis of Pseudomonas aeruginosa biofilms in cystic fibrosis
}

\author{
S.D. Aaron, D. Kottachchi, W.J. Ferris, K.L. Vandemheen, M.L. St. Denis, A. Plouffe, S.P. Doucette, \\ R. Saginur, F.T. Chan, K. Ramotar
}

Sputum versus bronchoscopy for diagnosis of Pseudomonas aeruginosa biofilms in cystic fibrosis. S.D. Aaron, D. Kottachchi, W.J. Ferris, K.L. Vandemheen, M.L. St. Denis, A. Plouffe, S.P. Doucette, R. Saginur, F.T. Chan, K. Ramotar. C) ERS Journals Ltd 2004

ABSTRACT: The present authors hypothesised that bronchoscopy with protected specimen brush may sample biofilm-forming bacteria adherent to the airway wall, whereas traditional sputum collection may not.

Pseudomonas aeruginosa obtained from sputum, bronchoalveolar lavage and protected brush, taken from the right upper lung bronchus of 12 adult patients with cystic fibrosis, were compared. Retrieved bacteria were genotyped, and grown in planktonic cultures and as biofilms, and susceptibilities to individual antibiotics and to antibiotic combinations were determined.

Bacterial cultures obtained using bronchoscopy did not yield any new strains of bacteria that were not also found in sputum. A total of 10 patients $(83 \%)$ had a single strain of $\boldsymbol{P}$. aeruginosa found using sputum, bronchoalveolar lavage and protected brush techniques, and two patients $(17 \%)$ had two strains recovered in sputum, but only one strain was recovered using bronchoscopic techniques. Susceptibility to single antibiotics and to antibiotic combinations were not different between planktonically or biofilmgrown bacteria derived from sputum, as compared to those obtained by bronchoalveolar lavage and protected brush.

In conclusion, sputum collection provides as much information as bronchoscopy for characterising the genotype and antibiotic susceptibility of chronic Pseudomonas aeruginosa infection in patients with stable cystic fibrosis.

Eur Respir J 2004; 24: 631-637.
Depts of Medicine and Pediatrics, and The Ottawa Health Research Institute, University of Ottawa, Ottawa, Ontario, Canada.

Correspondence: S.D. Aaron

The Ottawa Hospital, General Campus

501 Smyth Road, Box 211

Ottawa, Ontario

Canada

K1H 8L6

Fax: 16137396266

E-mail: saaron@ottawahospital.on.ca

Keywords: Bronchoscopy

cystic fibrosis

microbiology

Pseudomonas aeruginosa

Received: April 262004

Accepted after revision: June 112004

This study was supported by grants from Physician Services Inc., and the Ontario Thoracic Society, Ontario, Canada.
Pseudomonas aeruginosa is the most common pathogen that chronically infects patients with cystic fibrosis (CF) [1]. Recent evidence suggests that $P$. aeruginosa may actually grow as dense communities of exopolysaccharide-encapsulated biofilms adherent to $\mathrm{CF}$ patients' airways [2-4].

Patients with $\mathrm{CF}$ have sputum cultures taken regularly during clinically stable periods, and susceptibility results from these cultures are used to guide initial antibiotic therapy during exacerbations. One problem with relying on sputum samples to guide therapy is that biofilm bacteria may not be adequately sampled via sputum, since sputum expectoration only samples $P$. aeruginosa that have detached and dispersed from the airway biofilm $[5,6]$. In vitro studies have shown that biofilm-forming $P$. aeruginosa are significantly less susceptible to antibiotics compared to nonadherent forms $[3,7]$. However, studies have not previously been done to determine whether bacteria retrieved from sputum are more susceptible to antibiotics compared to bacteria retrieved directly from the airway biofilm.

Techniques for acquiring respiratory specimens that are more invasive, and potentially more accurate than sputum, have been investigated to determine their ability to detect bacterial flora present in patients' lungs. For example, semiquantitative broncheoalveolar lavage (BAL) has previously been evaluated for the detection of infections in the lower respiratory tract [8-11]. Bronchoscopy with protected specimen brush (PSB) has also been utilised to collect uncontaminated airway specimens for culture [12-14]. In contrast to BAL, where saline washings of bronchial secretions are aspirated for sampling, in PSB bronchoscopy the airway lumen is directly brushed to remove bacteria and the PSB is then retracted into its sterile sheath to minimise contamination with other airway secretions. Thus, it is possible that PSB may directly recover $P$. aeruginosa bacteria that have aggregated as biofilms and adhered to the surface of the airway.

The purpose of the present study was to determine whether the PSB can sample directly from the airway biofilm and, thus, more accurately represent the state of $P$. aeruginosa in CF patients' lungs. The authors wanted to determine whether bacteria adherent to the airways, retrieved via protected brush specimens, are genotypically or phenotypically different from the bacteria that are traditionally retrieved from sputum cultures. If this were the case, then this might suggest that bronchoscopy specimens are preferable over sputum for the accurate diagnosis of CF pulmonary infections.

\section{Materials and methods}

\section{Subjects}

Patients were eligible for this study if they were aged $>16$ yrs, had a confirmed diagnosis of $\mathrm{CF}$ with chronic $P$. aeruginosa 
infection, were able to spontaneously produce sputum, had a forced expiratory volume in one second (FEV1) $\geqslant 40 \%$ of predicted and were clinically stable and free of any oral or $i . v$. antibiotics for $>4$ weeks prior to study entry. The study was approved by the Ottawa Hospital Research Ethics Board and all subjects provided informed, written consent.

\section{Specimen collection}

Patients were required to spontaneously produce sputum immediately prior to bronchoscopy being performed. The same investigator performed the bronchoscopy on all 12 patients. Following expectoration of sputum, the upper airway was anaesthetised with $2 \%$ lidocaine spray. Patients were then sedated with intravenous fentanyl and diazepam, using an average dose of $50 \mu \mathrm{g}$ and $5 \mathrm{mg}$, respectively. Topical anaesthesia of the vocal cords and the trachea was achieved using $2 \%$ lidocaine, applied through the bronchoscope from above the cords. The bronchoscope (IT-160 and BF-200; Olympus Corporation, Hinode, Japan) was then introduced through the vocal cords and the tip was wedged in the anterior segment of the right upper lobe bronchus. Use of suction was avoided before wedging to keep the suction channel free of contamination. BAL was performed by instilling and aspirating five $20-\mathrm{mL}$ aliquots of sterile $0.9 \% \mathrm{NaCl}$ (CHASTRE et al. [12]). The BAL fluid was pooled and immediately placed on ice. Subsequent to the BAL procedure, the PSB (8115; Primed Instrument Incorp., Mississauga, ON, Canada) was introduced through the working channel of the bronchoscope and advanced into the anterior segment of the right upper lobe bronchus. After dislodging the distal catheter plug, the brush was applied to the wall of the subsegmental airway and agitated against the airway wall to procure lower airway secretions (CHASTRE et al. [13]). The brush was then cut and deposited into $1 \mathrm{~mL}$ of sterile saline and placed on ice.

\section{Specimen culture}

Sputum specimens were washed with an equal volume of sterile saline and the wash liquid discarded. An appropriate amount of sputum was then added to an equal amount of trypticase soy broth (TSB; Becton-Dickinson Company, Cockysville, MD, USA) and ground in a tissue grinder (Radnoti Glass, Monrovia, CA, USA). The specimens were further diluted $1: 10,1: 1,000$ and 1:100,000 with TSB, and $10 \mu \mathrm{L}$ of each dilution were inoculated onto trypticase soy agar with 5\% sheep blood agar (BA) and McConkey agar plates (PML Microbiologicals, Mississauga, ON, Canada).

BAL fluid was diluted 1:100 and 1:100,000 with TSB and $10 \mu \mathrm{L}$ of the undiluted specimen, and each of the two dilutions were inoculated onto media as described for sputum. PSB specimens were vortexed vigorously in $1 \mathrm{~mL}$ of sterile saline to assure complete release of all material from the brushes. Dilutions and inoculation of microbiological media was done as described for BAL fluids.

The BA and McConkey plates were incubated for 48-72 h at $35^{\circ} \mathrm{C}$ in ambient air. Colonies resembling Pseudomonas spp. were selected from either the BA or McConkey plates for identification and susceptibility testing. A total of five different colonies were randomly selected from each specimen type regardless of whether or not they represented the same or different morphological types. In this way, the cultures were deliberately over-sampled in order to maximise the recovery of as many different strain types as possible. All isolates selected for work-up were identified as $P$. aeruginosa by conventional microbiological methods, as described elsewhere $[15,16]$. All isolates were kept frozen at $-70^{\circ} \mathrm{C}$ in TSB containing 15\% glycerol (Sigma-Aldrich, St. Louis, MO, USA) until required for further testing.

\section{Pulsed-field gel electrophoresis-typing of isolates}

Molecular genotyping of $P$. aeruginosa isolates was carried out by pulsed-field gel electrophoresis (PFGE) as described by AARON et al. [17]. Restriction fragment profiles were visually compared and interpreted based on guidelines recommended by TeNOVER et al. [18]. Isolates with identical restriction fragment profiles were considered to represent a single strain. Isolates with restriction profiles that differed by $1-3$ fragments (bands) were considered to be closely related strains and were considered to have evolved from a single clone. Isolates with restriction profiles differing by four bands or more were considered to be different strains.

\section{Minimal inhibitory concentration determinations (planktonic growth)}

Minimal inhibitory concentrations (MICs) were determined for six anti-pseudomonal antibiotics: meropenem (AstraZeneca Pharmaceuticals, Wilmington, DE, USA), tobramycin (Sabex, Boucherville, QC, Canada), amikacin (Bristol laboratories, Montreal, QC, Canada), piperacillin (Wyeth-Ayerst, Montreal, QC, Canada), ciprofloxacin (Miles Inc., West Haven, CT, USA), and ceftazidime (GlaxoSmithKline, Mississauga, ON, Canada) for each isolate of $P$. aeruginosa. MICs were performed by broth microdilution and interpreted using National Committee for Clinical Laboratory Standards guidelines [19].

\section{Multiple combination bactericidal testing (planktonic growth)}

Multiple combination bactericidal testing (MCBT) was performed to assess the susceptibility of each isolate to multiple combinations of antibiotics, as previously described [20]. Ten antibiotics were tested against each $P$. aeruginosa in 94 different combinations. The combinations consisted of groupings of two or three of the following: azithromycin (Pfizer, New York, NY, USA), meropenem, ticarcillin-clavulanic acid (GlaxoSmithKline, Mississauga, ON, Canada), piperacillintazobactam (Wyeth-Ayerst), trimethoprim-sulfamethoxazole (GlaxoSmithKline), amikacin, ceftazidime, ciprofloxacin, chloramphenicol (Sigma-Aldrich, St. Louis, MO, USA) and tobramycin.

\section{Biofilm testing}

All of the $P$. aeruginosa isolates recovered from sputum, BAL and PSB specimens of the 12 patients were grown as biofilms using a modified Calgary Biofilm device [21]. Single and combination antibiotic susceptibility testing of $P$. aeruginosa, grown as biofilms, has been previously validated and shown to be reproducible [7, 21].

Briefly, for biofilm growth, the isolates were grown for $24 \mathrm{~h}$ adherent to polystyrene pegs that sat in standard 96-well plates (Nalge Nunc International, Roskilde, Denmark) at $35^{\circ} \mathrm{C}$ in ambient air. A rocking table was used to produce shear forces across each peg, resulting in the formation of equivalent biofilms at each peg site. The current authors have previously shown by electron microscopy that this method results in biofilm growth on the pegs [7]. The biofilm culture was then transferred to a standard 96-well plate in which dilutions of antibiotics had been prepared and then incubated 
overnight at $35^{\circ} \mathrm{C}$ in ambient air. The MIC for the biofilm was determined to be the concentration of antibiotic at which biofilm growth (absence of turbidity in the well) was inhibited. The biofilm was then removed from the pegs by sonication and the viability of the bacterial biofilm (minimum biofilm eradication concentration) was assessed by plate counts [7].

\section{Statistical analysis}

A generalised estimating equations (GEE) model was used to compare the sputum isolates to the BAL and PSB isolates to determine whether there were any significant differences in their MIC values. The GEE model is an extension of the generalised linear model and allows for correlation between individuals and does not require a normal distribution of data. Results were analysed modelling antibiotics individually, and also treating antibiotic type as a covariate to compare susceptibility of sputum, BAL and PSB isolates across all six antibiotics. MCBT results were analysed with paired t-tests to determine whether there were significant differences between sputum and BAL, and sputum and PSB results. A p-value $<0.05$ was considered statistically significant.

\section{Results}

\section{Study population}

The study population consisted of nine male and three female patients with CF whose average age was $29 \mathrm{yrs}$. All patients had been chronically infected with mucoid
$P$. aeruginosa isolates for an average duration of $8 \pm 5 \mathrm{yrs}$ (range: 3-19 yrs). Relevant patient characteristics are given in table 1 . All 12 study patients tolerated the bronchoscopy well without complications.

Table 1.-Baseline characteristics of cystic fibrosis (CF) patients

\begin{tabular}{lc}
\hline Characteristic & \\
\hline Age yrs & $29.3 \pm 8.0$ \\
Male sex & $9(75)$ \\
FEV1 & \\
L & $2.41 \pm 0.80$ \\
$\%$ pred & $64.6 \pm 21.2$ \\
FVC & \\
L & $3.86 \pm 0.76$ \\
\% pred & $85.6 \pm 19.8$ \\
Duration of $P$. aeruginosa infection yrs & $8.2 \pm 5.1$ \\
Pancreatic insufficient & $11(92)$ \\
Diabetes & $0(0)$ \\
Liver disease & $1(8)$ \\
Medications for CF & $8(67)$ \\
Bronchodilators & $5(42)$ \\
Inhaled corticosteroids & $6(50)$ \\
Inhaled tobramycin & $12(100)$ \\
Vitamins & $11(12)$ \\
Pancreatic enzymes & \\
\hline
\end{tabular}

Data are presented as mean \pm SD or $n(\%), n=12$. FEV1: forced expiratory volume in one second; FVC: forced vital capacity; P. aeruginosa: Pseudomonas aeruginosa.

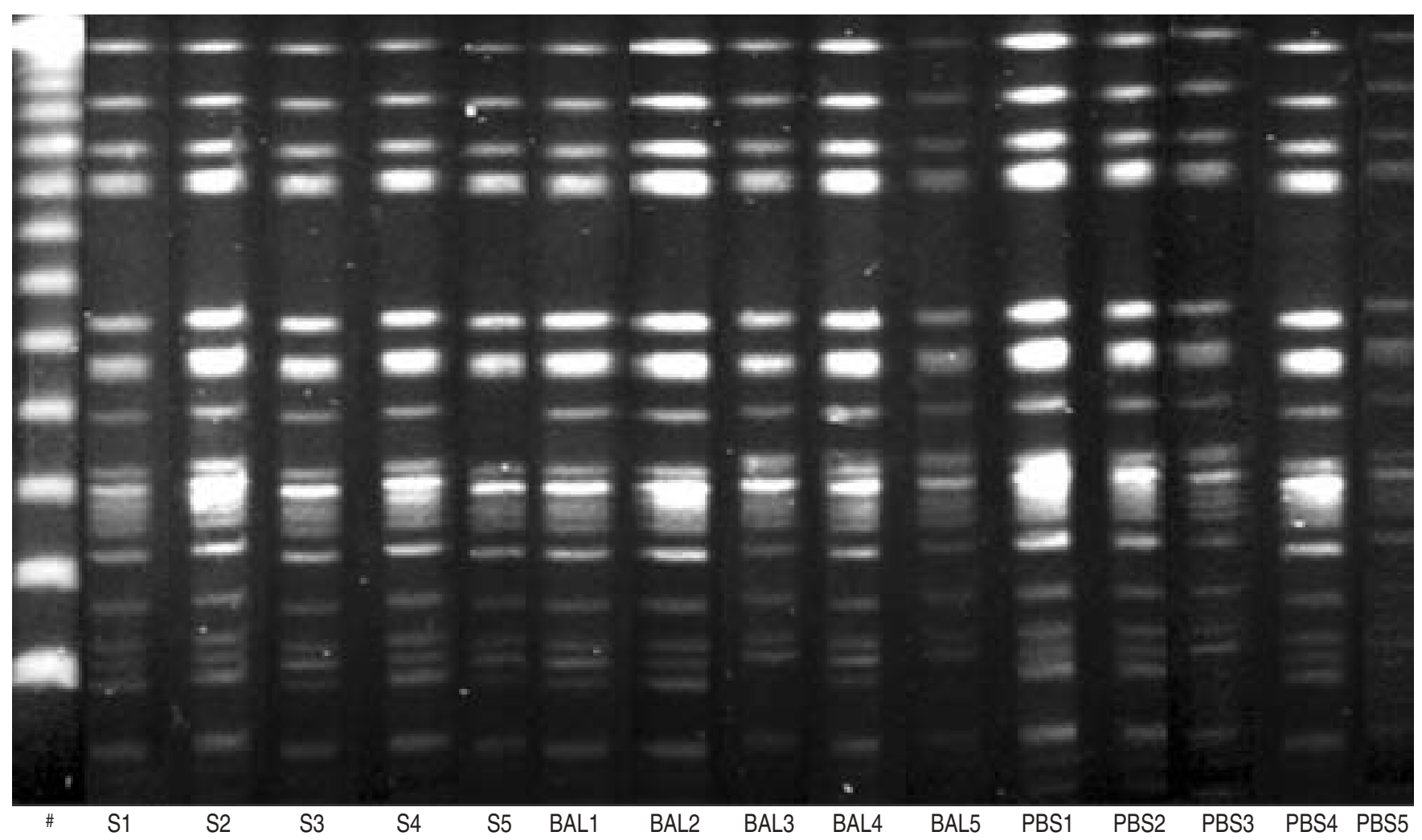

Fig. 1.-Pulsed-field gel electrophoresis (PFGE) of Pseudomonas aeruginosa isolates from patient 4. The genomic DNA for the 15 P. aeruginosa isolates from each patient (five for each of the three sampling techniques) were analysed by PFGE following restriction digestion with SpeI (restriction endonuclease). All the isolates from this patient were either derived from a single strain or a closely related strain of $P$. aeruginosa, since none of the isolates differed by more than three restriction bands. S: sputum; BAL: bronchoalveolar lavage; PSB: protected specimen brush. ${ }^{\#}$ : 48.5-kb DNA ladder. 


\section{Quantitative cultures}

Quantitative culture analysis of the 15 planktonically grown isolates (five sputum, five BAL and five PSB) retrieved from each of the $12 \mathrm{CF}$ patients revealed that they all had $\geqslant 1,000,000 \mathrm{cfu} \cdot \mathrm{mL}^{-1}$ of $P$. aeruginosa present in their samples. This amount of bacteria is well above the $10,000 \mathrm{cfu} \cdot \mathrm{mL}^{-1}$ cut-off currently mandated for accurately diagnosing lung infections with PSB and BAL $[11,12]$.

\section{Bacterial genotyping}

Bacterial cultures obtained using bronchoscopy did not yield any new strains of bacteria that were not also found in sputum. PFGE revealed that 10 patients $(83 \%)$ had a single strain of $P$. aeruginosa found in all sputum, BAL and PBS cultures (fig. 1). Two patients $(17 \%)$ had two strains of $P$. aeruginosa recovered from sputum, but only one strain was recovered from BAL and PSB (fig. 2).

Table 2 summarises the genotypes of the patients' isolates. A total of five patients (patients 1, 3, 5, 7 and 11) were discovered to have a common ' $\mathrm{A}$ ' strain of $P$. aeruginosa growing from sputum.

\section{Single-antibiotic susceptibilities}

Table 3 summarises the single antibiotic MIC data as $\log _{2}$ values for the planktonically grown isolates. Antibiotic
Table 2. - Summary of Pseudomonas aeruginosa strains

\begin{tabular}{lccc}
\hline Patient & \multicolumn{2}{c}{ Genotype of $P$. aeruginosa retrieved } \\
\cline { 2 - 4 } & Sputum & BAL & PSB \\
\hline 1 & $\mathrm{~A}$ & $\mathrm{~A}$ & $\mathrm{~A}$ \\
2 & $\mathrm{~F} / \mathrm{G}^{\#}$ & $\mathrm{~F}$ & $\mathrm{~F}$ \\
3 & $\mathrm{~A}$ & $\mathrm{~A}$ & $\mathrm{~A}$ \\
4 & $\mathrm{H}$ & $\mathrm{H}$ & $\mathrm{H}$ \\
5 & $\mathrm{~A}$ & $\mathrm{~A}$ & $\mathrm{~A}$ \\
6 & $\mathrm{~J}$ & $\mathrm{~J}$ & $\mathrm{~A}$ \\
7 & $\mathrm{~A}$ & $\mathrm{D}$ & $\mathrm{A}$ \\
8 & $\mathrm{D}$ & $\mathrm{B}$ & $\mathrm{D}$ \\
9 & $\mathrm{~B}$ & $\mathrm{C}$ & $\mathrm{B}$ \\
10 & $\mathrm{C}$ & $\mathrm{A}$ & $\mathrm{C}$ \\
11 & $\mathrm{~A} / \mathrm{D}^{\#}$ & $\mathrm{E}$ & $\mathrm{A}$ \\
12 & $\mathrm{E}$ & $\mathrm{E}$ \\
\hline
\end{tabular}

Genotypes are arbitrarily designated an alphabetic letter based on their restriction fragment profiles. A single letter indicates that all five isolates for that sampling technique yielded a single strain of bacteria Patients 2 and 11 had two strains of $P$. aeruginosa bacteria present in sputum. Five patients (patients 1, 3, 5, 7, 11) shared a common "A" strain of $P$. aeruginosa. BAL: bronchoalveolar lavage; PSB: protected specimen brush. ${ }^{\#}$ : patients with two strains of $P$. aeruginosa in sputum.

susceptibilities were independent of the source of the $P$. aeruginos $a$ isolate $(\mathrm{p}=0.76$ and $\mathrm{p}=0.65$ for the comparison of sputum and BAL MICs, and sputum and PSB MICs, respectively). There were no significant differences in antibiotic susceptibility between the three sampling techniques for

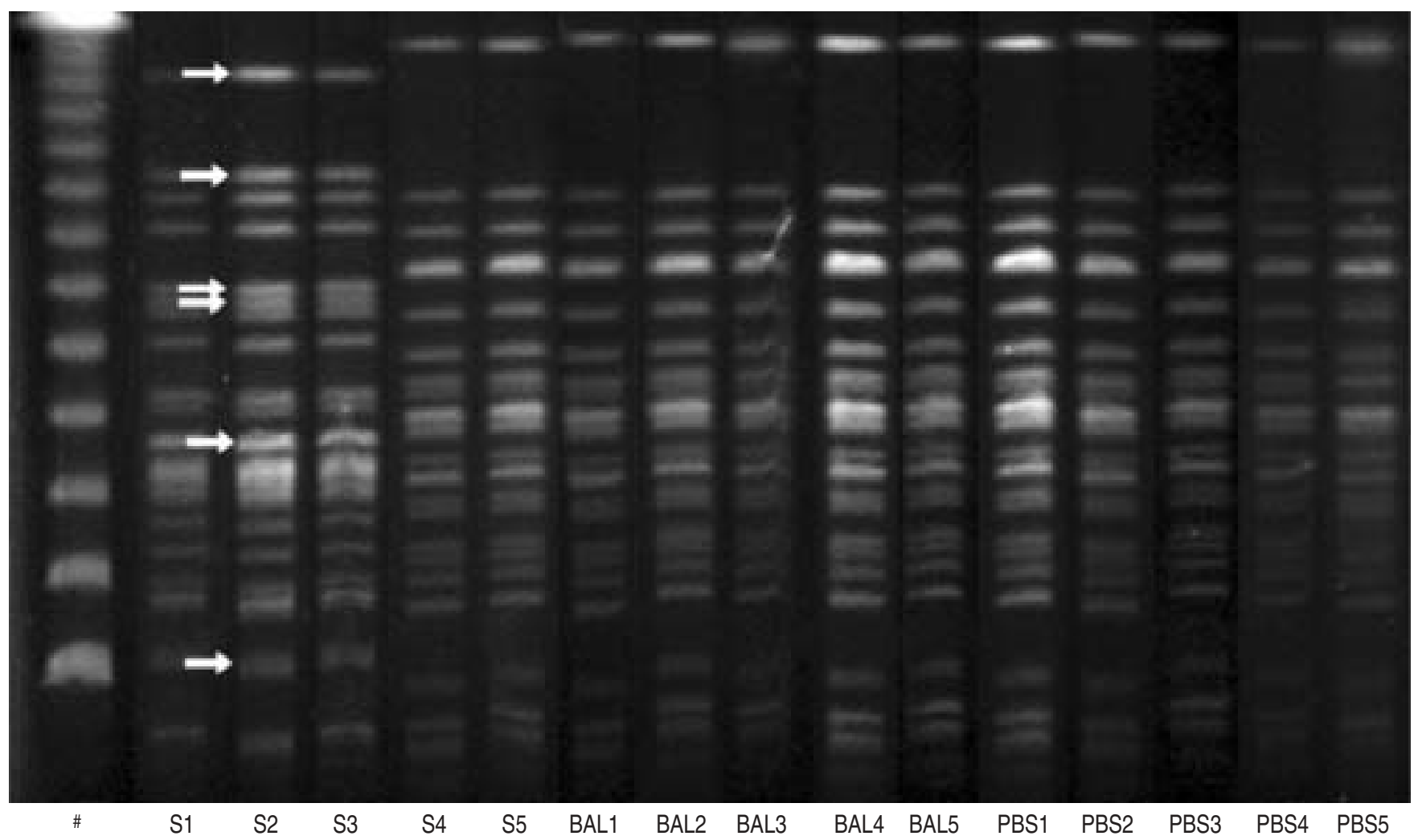

Fig. 2. - Pulsed-field gel electrophoresis (PFGE) of Pseudomonas aeruginosa isolates from patient 2. The genomic DNA for the 15 P. aeruginosa isolates from each patient (five for each of the three sampling techniques) were analysed by PFGE following restriction digestion with SpeI (restriction endonuclease). Isolate profiles in lanes 5-16 are derived from the same strain of $P$. aeruginosa. However, isolates 1 , 2 and 3 from sputum are a different strain of $P$. aeruginosa than the other isolates (see white arrows for illustrative examples of different bands). S: sputum; BAL: bronchoalveolar lavage; PSB: protected specimen brush. ${ }^{\#}$ : $48.5-\mathrm{kb}$ DNA ladder. 
Table 3.-Single antibiotic susceptibility results presented as minimal inhibitory concentration (MIC) log values for the planktonically grown isolates obtained from cystic fibrosis patients

\begin{tabular}{|c|c|c|c|c|c|c|c|}
\hline \multirow[t]{3}{*}{ Antibiotic } & \multicolumn{3}{|c|}{ MICs for isolates } & \multicolumn{4}{|c|}{ p-values } \\
\hline & \multirow[t]{2}{*}{ Sputum } & \multirow[t]{2}{*}{ BAL } & \multirow[t]{2}{*}{ PSB } & \multicolumn{2}{|c|}{ Sputum versus BAL } & \multicolumn{2}{|c|}{ Sputum versus PSB } \\
\hline & & & & IA & $\mathrm{AA}$ & IA & AA \\
\hline Tobramycin & $1.82 \pm 1.50$ & $1.42 \pm 1.50$ & $1.47 \pm 1.49$ & 0.07 & 0.76 & 0.09 & 0.65 \\
\hline Meropenem & $-1.50 \pm 1.74$ & $-1.67 \pm 1.78$ & $-1.15 \pm 2.38$ & 0.41 & & 0.31 & \\
\hline Amikacin & $4.78 \pm 1.19$ & $4.77 \pm 1.48$ & $5.00 \pm 1.46$ & 0.94 & & 0.49 & \\
\hline Ceftazidime & $3.28 \pm 1.68$ & $3.90 \pm 2.25$ & $3.50 \pm 2.53$ & 0.04 & & 0.62 & \\
\hline Piperacillin & $5.15 \pm 2.31$ & $5.30 \pm 2.26$ & $5.28 \pm 2.47$ & 0.55 & & 0.75 & \\
\hline Ciprofloxacin & $-0.67 \pm 2.10$ & $-0.53 \pm 1.89$ & $-0.53 \pm 2.10$ & 0.53 & & 0.45 & \\
\hline
\end{tabular}

MIC values (mean \pm SD) are shown as $\log _{2}$ values of the dilution used. $n=12$. Comparative $p$-values are derived from the generalised estimating equations model. BAL: bronchoalveolar lavage; PSB: protected specimen brush; IA: individual antibiotics; AA: all antibiotics.

all six antibiotics tested. The one exception was ceftazidime, where isolates obtained via BAL were less susceptible to ceftazidime than those obtained from sputum $(\mathrm{p}=0.04)$.

Table 4 summarises the single antibiotic MIC data as $\log _{2}$

Table 4. - Single antibiotic susceptibility results presented as minimal inhibitory concentration (MIC) $\log _{2}$ values for the biofilm grown isolates obtained from cystic fibrosis patients

\begin{tabular}{lcccccc}
\hline Antibiotic & \multicolumn{3}{c}{ MICs for isolates } & & \multicolumn{2}{c}{ p-values } \\
\cline { 2 - 4 } \cline { 6 - 7 } & Sputum & BAL & PSB & & $\begin{array}{c}\text { Sputum } \\
\text { versus } \\
\text { BAL }\end{array}$ & $\begin{array}{c}\text { Sputum } \\
\text { versus } \\
\text { PSB }\end{array}$ \\
\hline Tobramycin & $6.0 \pm 0.0$ & $6.0 \pm 0.0$ & $6.0 \pm 0.0$ & 0.99 & 0.99 \\
Meropenem & $5.0 \pm 0.0$ & $5.0 \pm 0.0$ & $5.0 \pm 0.0$ & & 0.99 & 0.99 \\
Amikacin & $7.0 \pm 0.0$ & $7.0 \pm 0.0$ & $7.0 \pm 0.0$ & & 0.99 & 0.99 \\
Ceftazidime & $8.0 \pm 0.0$ & $8.0 \pm 0.0$ & $8.0 \pm 0.0$ & & 0.99 & 0.99 \\
Piperacillin & $8.0 \pm 0.0$ & $8.0 \pm 0.0$ & $8.0 \pm 0.0$ & & 0.99 & 0.99 \\
Ciprofloxacin & $3.93 \pm 0.23$ & $3.87 \pm 0.21$ & $3.80 \pm 0.4$ & & 0.37 & 0.10 \\
\hline
\end{tabular}

MIC values (mean $\pm \mathrm{SD}$ ) were obtained from paired t-test analysis and are shown as $\log _{2}$ values of the dilution used. $\mathrm{n}=12$. Comparative p-values are derived from the generalised estimating equations model. BAL: bronchoalveolar lavage; PSB: protected specimen brush. values for the isolates grown as biofilms. For all these isolates, there were no significant differences in susceptibility between BAL- and PSB-acquired samples in comparison to the isolates from sputum. Minimum biofilm eradication antibiotic concentrations were even higher than the biofilm MICs and they similarly showed no differences between sputum, BAL or PSB-derived isolates (data not shown).

\section{Combination antibiotic susceptibilities}

Table 5 summarises the MCBT data. A total of 94 double and triple antibiotic combinations were assessed for bactericidal activity against each isolate. There were no significant differences observed between the number of susceptible antibiotic combinations for the planktonically grown sputum isolates (mean: $75.9 \pm 7.5$ ) compared to BAL isolates (mean: $76.2 \pm 8.6, \mathrm{p}=0.80$ ) or PSB isolates (mean: 76.1 \pm 11.0 , $\mathrm{p}=0.89)$.

Similar results were seen for biofilm-grown isolates. Biofilm-grown isolates were less susceptible to antibiotic combinations than planktonic-grown isolates. However, there were no significant differences observed when comparing the number of susceptible antibiotic combinations for the biofilmgrown sputum isolates (mean: $31.0 \pm 9.0$ ) compared to BAL

Table 5. - Multiple combination bactericidal testing results

Patient Mean number of bactericidal combinations for the five isolates from each patient

\begin{tabular}{|c|c|c|c|c|c|c|}
\hline & \multicolumn{3}{|c|}{ Planktonic } & \multicolumn{3}{|c|}{ Biofilm } \\
\hline & Sputum & BAL & PSB & Sputum & BAL & PSB \\
\hline 1 & 81 & 81 & 83 & 21 & 13 & 24 \\
\hline 2 & 84 & 88 & 87 & 24 & 30 & 25 \\
\hline 3 & 76 & 69 & 73 & 23 & 6 & 22 \\
\hline 4 & 61 & 60 & 72 & 35 & 23 & 40 \\
\hline 5 & 63 & 70 & 54 & 18 & 36 & 21 \\
\hline 6 & 83 & 85 & 85 & 48 & 26 & 38 \\
\hline 7 & 71 & 71 & 71 & 30 & 37 & 40 \\
\hline 8 & 80 & 81 & 91 & 38 & 45 & 41 \\
\hline 9 & 75 & 69 & 64 & 24 & 24 & 13 \\
\hline 10 & 78 & 85 & 85 & 37 & 38 & 31 \\
\hline 11 & 76 & 74 & 66 & 38 & 34 & 30 \\
\hline 12 & 82 & 82 & 83 & 35 & 17 & 11 \\
\hline Mean \pm SD & $75.9 \pm 7.5^{\#}$ & $76.2 \pm 8.6$ & $76.1 \pm 11.0$ & $31.0 \pm 9.0^{+}$ & $27.5 \pm 11.4$ & $28.1 \pm 10.4^{\S}$ \\
\hline
\end{tabular}

Each cell represents the mean number of susceptible drug combinations (out of a total of 94) for the five isolates from each patient (five from sputum, five from bronchoalveolar lavage (BAL) and five from protected specimen brush (PSB)). Paired t-tests were used to compare differences between the mean number of susceptible drug combinations between sputum and BAL, and sputum and PSB. ${ }^{\#}: \mathrm{p}=0.80$, versus planktonic BAL; ${ }^{\uparrow}$ : $=0.89$, versus planktonic sputum; ${ }^{+}: \mathrm{p}=0.34$, versus biofilm BAL; ${ }^{\S}: \mathrm{p}=0.29$, versus biofilm sputum. 
isolates (mean: $27.5 \pm 11.4, \mathrm{p}=0.34$ ) or PSB isolates (mean: $28.1 \pm 10.4, \mathrm{p}=0.29)$.

\section{Discussion}

Prior to this study, no previous investigations have been conducted to explore whether bronchoscopy can obtain cultures of bacteria directly from in vivo biofilms and whether these bacteria are genetically different or more resistant to antibiotics than those obtained from sputum cultures. In this group of 12 stable CF patients, neither the PSB nor the BAL isolates provided any additional relevant information regarding strain type and antibiotic sensitivity of the $P$. aeruginosa bacteria in comparison to those samples isolated from sputum.

Bronchoscopy specimens did not yield any $P$. aeruginosa strains that were not found in sputum. A possible explanation for this is that bronchoscopy sampling was limited to the right upper lobe only in the present study to avoid contamination of the sample port from suctioning multiple sites. Expectorated sputum samples a greater proportion of the airways bilaterally and may, potentially, provide a more accurate picture of the flora present. Alternatively, it is possible that the additional $P$. aeruginosa strains recovered in the sputum from two patients, but not recovered from their right upper lobes using bronchoscopy, may have originated from these patients' upper airways or sinuses.

A total of 10 out of 12 patients had a single distinct strain of $P$. aeruginosa in all their isolates, and sputum cultures from only two patients produced two distinct strains. The presented results are consistent with those generated from previous investigations of $P$. aeruginosa in chronically infected $\mathrm{CF}$ patients $[17,22,23]$. These studies have all reported that, over time, most adult $\mathrm{CF}$ patients tend to harbour a single clone or subclone of $P$. aeruginosa during periods of clinical stability, and that the same bacterial clone retrieved during periods of clinical stability is also retrieved during pulmonary exacerbations [17].

The current study used standard serial dilution microbiological techniques to isolate, identify and quantify organisms. Serial dilution techniques may not identify organisms that are present only in very low numbers. However, isolates were deliberately over-sampled from the culture plates in order to maximise the recovery of as many different strain types as possible.

A total of five out of the 12 patients shared the same strain of $P$. aeruginosa in all specimen types. This indicates that there may be a strain of $P$. aeruginosa circulating through the clinic at our centre causing infection in a proportion of our $\mathrm{CF}$ patients. This observation is consistent with the findings of several other studies [24-28]. These studies have all provided convincing evidence that cross-infection in $\mathrm{CF}$ patients may exist and imply that limited shared contact in a clinic setting may be sufficient to transfer strain types between CF patients. ARMSTRONG et al. [28] and Jones et al. [29] both suggested that the most likely mode of spread was direct patient-to-patient contact.

When the single antibiotic susceptibilities for $P$. aeruginosa obtained from sputum were compared to those obtained by bronchoscopy, no significant differences in MIC values were observed. Similar results were found when comparing susceptibilities of isolates to antibiotic combinations. Based on these observations, isolates obtained through bronchoscopy techniques would not provide any additional advantages over sputum isolates for assessing antibiotic susceptibility of $P$. aeruginosa in $\mathrm{CF}$ patients.

BAUGHMAN et al. [11] published a study in which sputum culture results were compared to BAL semiquantitative culture results from 28 bronchoscopies done in 11 adult patients with $\mathrm{CF}$. They showed that in 11 instances BAL was able to either detect a new pathogen, or detect an additional pathogen that sputum cultures did not. This led to an alteration in therapy in $48 \%$ of the patients investigated. The major difference in the study by BAUGHMAN et al. [11], in comparison to the current report, was that there were inadequate sputum cultures for comparison in 11 out of their 28 BAL procedures. Since many of their patients could not produce sputum, bronchoscopy revealed pathogens not otherwise identified.

The present study was restricted to clinically stable patients. The authors chose to conduct this study in clinically stable patients due to safety concerns and also to mimic clinical practice. Patients with $\mathrm{CF}$ have sputum cultures taken regularly during clinically stable periods and susceptibility results from these cultures are used to guide initial antibiotic therapy during exacerbations. A previous study [17] has confirmed that the vast majority of $P$. aeruginosa isolates retrieved during exacerbations are identical to those retrieved during clinical stability. Thus, the patients presented here are representative of those who would benefit the most from an alternative, and potentially more accurate, airway sampling technique during clinically stable periods to direct their therapy when they become ill.

Antibiotic susceptibility testing of Pseudomonas aeruginosa isolated from sputum remains a requirement for directing therapy in chronically infected cystic fibrosis patients. However, antibiotic administration hardly ever eliminates carriage of this bacterium, but rather reduces symptoms of pulmonary exacerbations. Given the current findings, the authors feel that the failure of antibiotic therapy to sterilise the adult cystic fibrosis airway is not due to any inherent limitation in the ability of sputum to adequately sample the airway flora. In conclusion, two bronchoscopic techniques (bronchoalveolar lavage and protected specimen brush) failed to show any benefit over sputum in sampling the type of Pseudomonas aeruginosa infecting the lungs of stable cystic fibrosis patients.

\section{References}

1. Fick RB Jr. Pathogenesis of the pseudomonas lung lesion in cystic fibrosis. Chest 1989; 96: 158-164.

2. Singh PK, Parsek MR, Greenberg EP, Welsh MJ. A Component of innate immunity prevents baceterial biofilm development. Nature 2002; 417: 552-555.

3. Drenkard E, Ausubel FM. Pseudomonas biofilm formation and antibiotic resistance are linked to phenotypic variation. Nature 2002; 416: 740-743.

4. Singh PK, Schaefer AL, Parsek MR, Moninger TO, Welsh MJ, Greenberg EP. Quorum-sensing signals indicate that cystic fibrosis lungs are infected with bacterial biofilms. Nature 2000; 407: 762-764.

5. Costerton JW. Introduction to biofilm. Int $J$ Antimicrob Agents 1999; 11: 217-221.

6. Costerton JW, Stewart PS, Greenberg EP. Bacterial biofilms: a common cause of persistent infections. Science 1999; 284: $1318-1322$.

7. Aaron SD, Ferris W, Ramotar K, Vandemheen K, Chan F, Saginur R. Single and combination antibiotic susceptibilities of planktonic, adherent and biofilm-grown Pseudomonas aeruginosa cultured from CF sputum. J Clin Microbiology 2002; 40: 4172-4179.

8. Kahn FW, Jones JM. Diagnosing bacterial respiratory infection by bronchoalveolar lavage. J Infect Dis 1987; 155: 862-869.

9. Thorpe JE, Baughman RP, Frame PT, Wesseler TA, Staneck 
JL. Bronchoalveolar lavage for diagnosing acute bacterial pneumonia. J Infect Dis 1987; 155: 855-861.

10. Konstan MW, Hilliard KA, Norvell TM, Berger M. Bronchoalveolar lavage findings in cystic fibrosis patients with stable, clinically mild lung disease suggest ongoing infection and inflammation. Am $J$ Respir Crit Care Med 1994; 150: 448-454.

11. Baughman RP, Keeton DA, Perez C, Wilmott RW. Use of bronchoalveolar lavage semiquantitative cultures in cystic fibrosis. Am J Respir Crit Care Med 1997; 156: 286-291.

12. Chastre J, Fagon J-Y, Bornet-Lesco M, et al. Evaluation of bronchoscopic techniques for the diagnosis of nosocomial pneumonia. Am J Respir Crit Care Med 1995; 152: 231-240.

13. Chastre J, Viau F, Brun P, et al. Prospective evaluation of the protected specimen brush for the diagnosis of pulmonary infections in ventilated patients. Am Rev Respir Dis 1984; 130: 924-929.

14. Higuchi JH, Coalson JJ, Johanson WG Jr. Bacteriologic diagnosis of nosocial pneumonia in primates. Usefulness of the protected specimen brush. Am Rev Respir Dis 1982; 125: 53-57.

15. Gilligan PH. Microbiology of airway disease in patients with cystic fibrosis. Clin Microbiology Rev 1991; 4: 35-51.

16. Miller MB, Gilligan PH. Laboratory aspects of management of chronic pulmonary infections in patients with cystic fibrosis. J Clin Microbiol 2003; 41: 4009-4015.

17. Aaron SD, Ramotar K, Ferris W, et al. Adult cystic fibrosis exacerbations and new strains of Pseudomonas aeruginosa. Am J Respir Crit Care Med 2004; 169: 811-815.

18. Tenover FC, Arbeit RD, Goering RV. Interpreting chromosomal DNA restriction patterns produced by pulse-field gel electrophoresis: criteria for bacterial stain typing. $J$ Clin Microbiol 1995; 33: 2233-2239.

19. Methods for dilution antimicrobial susceptibility tests for bacteria that grow aerobically; approved standards. National Committee for Clinical Laboratory Standards (NCCLS). Wayne PA, NCCLS, 2003.

20. Aaron SD, Ferris W, Henry DA, Speert DP, MacDonald
NE. Multiple combination bactericidal antibiotic testing for patients with cystic fibrosis infected with Burkholderia cepacia. Am J Respir Crit Care Med 2000; 161: 1206-1212.

21. Ceri H, Olson ME, Stremick C, Read RR, Morck D, Buret A. The Calgary Biofilm Device: new technology for rapid determination of antibiotic susceptibilities of bacterial biofilms. J Clin Microbiol 1999; 37: 1771-1776.

22. Breitenstein S, Walter S, Bobhammer J, Romling U, Tummler B. Direct sputum analysis of Pseudomonas aeruginosa macrorestriction fragment genotypes in patients with cystic fibrosis. Med Microbiol Immunol 1997; 186: 93-99.

23. Struelens MJ, Schwam V, Delpano A, Baran D. Genome macrorestriction analysis of diversity and variability of Pseudomonas aeruginosa strains infecting cystic fibrosis patients. J Clin Microbiol 1993; 31: 2320-2326.

24. Cheng K, Smyth RL, Govan JRW, et al. Spread of $\beta$-lactamresistant Pseudomonas aeruginosa in a cystic fibrosis clinic. Lancet 1996; 348: 639-642.

25. Jones AM, Govan JRW, Doherty CJ, et al. Spread of a multiresistant strain of Pseudomonas aeruginosa in an adult cystic fibrosis clinic. Lancet 2001; 358: 557-558.

26. McCullum SJ, Corkill J, Gallagher M, Ledson MJ, Hart CA, Walshaw MJ. Superinfection with a transmissible strain of Pseudomonas aeruginosa in adults with cystic fibrosis chronically colonised by $P$ aeruginosa. Lancet 2001; 358: $558-560$.

27. Anthony M, Rose B, Beard-Pegler M, et al. Genetic analysis of Pseudomonas aeruginosa isolates from the sputa of Australian adult cystic fibrosis patients. J Clin Microbiol 2002; 40: 2772-2778.

28. Armstrong DS, Nixon GM, Carzino R, et al. Detection of a widespread clone of Pseudomonas aeruginosa in a pediatric cystic fibrosis clinic. Am J Resp Crit Care Med 2002; 166: 983-987.

29. Jones AM, Webb AK, Govan JR, Hart CA, Walshaw MJ. Pseudomonas aeruginosa cross-contamination in cystic fibrosis. Lancet 2002; 359: 527-528. 